\title{
Awareness of nonalcoholic steatohepatitis and associated practice patterns of primary care physicians and specialists
}

\author{
Susan Polanco-Briceno ${ }^{*}$ (D) Daniel Glass, Mark Stuntz and Alexis Caze
}

\begin{abstract}
Background: The hepatic manifestation of metabolic syndrome is nonalcoholic fatty liver disease. Patients with nonalcoholic steatohepatitis, the progressive form of nonalcoholic fatty liver disease, have increased risk of fibrosis, cirrhosis and end-stage liver disease. Estimates of prevalence in the United States range from 20-30 \% for nonalcoholic fatty liver disease and 2-5\% for nonalcoholic steatohepatitis; however, physician awareness of these diseases is limited. The purpose of this study was to determine the current level of physician awareness and practices in the diagnosis and management of nonalcoholic fatty liver disease and nonalcoholic steatohepatitis within the United States.
\end{abstract}

Methods: Physicians were asked to participate in an online, 35-question survey about their awareness of various liver conditions and current practices.

Results: Of the 302 responding physicians, 152 were primary care physicians, and 150 were specialists (comprised of gastroenterologists and hepatologists). More specialists than primary care physicians reported that they were aware of the differences between nonalcoholic fatty liver disease and nonalcoholic steatohepatitis $(p<0.001)$ and that they routinely screened for nonalcoholic fatty liver disease $(p<0.001)$ and nonalcoholic steatohepatitis $(p<0.001)$. Almost half of the responding primary care physicians reported being unfamiliar with the nonalcoholic fatty liver disease and nonalcoholic steatohepatitis differences even though they were aware of both, yet $58 \%$ of those primary care physicians were treating patients with nonalcoholic fatty liver disease and/or nonalcoholic steatohepatitis. In addition, those primary care physicians who reported being unfamiliar with nonalcoholic steatohepatitis were treating an average of 3.7 patients and reported being as likely as familiar primary care physicians to treat new patients with nonalcoholic steatohepatitis. More than half of the specialists used noninvasive diagnostic test to confirm nonalcoholic steatohepatitis, and $10 \%$ of the specialists reported treating patients with drugs not recommended by the current guidelines.

Conclusions: Despite reporting they were not familiar with nonalcoholic steatohepatitis, primary care physicians reported they would likely continue to diagnose and manage patients with nonalcoholic steatohepatitis; therefore, more physician education on the recent practice guideline for nonalcoholic fatty liver disease and nonalcoholic steatohepatitis is needed.

Keywords: Nonalcoholic fatty liver disease, Nonalcoholic steatohepatitis, Awareness, Practice guideline, Diagnosis, Disease management, Primary care physicians

\section{Background}

Non-alcoholic fatty liver disease (NAFLD) is increasingly recognized as a major health burden in developed

*Correspondence: spolanco-briceno@deerfield.com

Deerfield Institute, 780 3rd Ave., 36th Floor, New York, NY 10017, USA countries. It includes a spectrum of liver damage ranging from simple steatosis to nonalcoholic steatohepatitis (NASH), advanced fibrosis, and rarely, progression to cirrhosis [1]. In 2013, NASH became the second leading etiology of chronic liver disease among new liver transplant waitlist registrations [2]. A systematic review estimated 
the prevalence in the United States is about $33 \%$ for NAFLD and $2-5 \%$ for NASH [3]. The most recent diagnosis and management guidelines for NAFLD and NASH were published in 2012 [1]. Although it is widely accepted that a liver biopsy is the gold standard for the detection of NASH, but that it is impractical, unnecessary and cost prohibitive to perform a liver biopsy among all patients with NAFLD [4], no consensus has been reached in identifying specific biomarkers as an alternative for the diagnosing and monitoring of NAFLD-related liver disease [5]. Thus, clinical practice patterns in the diagnosing of NASH frequently diverge from published practice guidelines [6]. The lack of practical guidelines is particularly important among primary care physicians given the association of nonalcoholic fatty liver disease (NAFLD) to conditions frequently seen by these physicians, such as obesity and type 2 diabetes, and overall metabolic risk factors $[1,7,8]$. Indeed, existing data suggest there is limited awareness among general practitioners of NAFLD and its progressive form nonalcoholic steatohepatitis (NASH) [9-12].

In 2012, the American Association for the Study of Liver Disease, American College of Gastroenterology and the American Gastroenterological Association published a practice guideline for NAFLD and NASH [1]. More recently, two abstracts reported on the current practices of diagnosing and treating NASH and NAFLD using the guideline [13, 14]. Even though liver biopsy is the gold standard for diagnosing $\mathrm{NASH}$, responding physicians indicated that $39 \%$ of their NASH-diagnosed patients had not had a liver biopsy [13], and fewer than $25 \%$ of physician respondents said they routinely performed a liver biopsy to diagnose NASH [14]. In addition, $53 \%$ of physicians mentioned prescribing vitamin $\mathrm{E}$ for $\mathrm{NASH}$ treatment [14], even though the guideline indicates it should not be used without a liver biopsy [1], and $50 \%$ mentioned prescribing metformin for some patients specifically to treat NASH [13] while the guidelines do not recommend its use for treating NASH [1].

Familiarity with position statements and/or the practice guideline for NAFLD or NASH may also affect NASH management. Kallman et al. [15] found that PCPs were less likely than gastroenterologists or hepatologists to be familiar with the American Association for the Study of Liver Diseases position paper on NAFLD and were more likely to defer to the American Association of Family Practice guideline. This study, however, was completed prior to the release of the practice guideline for the diagnosis and management of NAFLD in 2012 [1], and no studies have yet to be reported on whether knowledge of the practice guideline has improved confidence in treating these patients.
The prevalence of NAFLD and NASH also varies depending on the method of detection and population being studied. Using data from the United States Third National Health and Nutrition Examination Survey (NHANES III) study, overall prevalence of NAFLD excluding NASH was $16.4 \%$ and the prevalence of NASH was $3.1 \%$ [16]; but, the authors diagnosed NASH based on increased liver enzymes in the presence of moderateto-severe hepatic steatosis in the absence of antibodies to hepatitis B and C and without evidence of iron overload [16]. A systematic review estimated the prevalence in the United States is about $33 \%$ for NAFLD and 2-5\% for NASH [3]. However, these estimates were based on studies with small cohorts. In a prospective study using ultrasound as an initial screening tool and biopsy confirmation, Williams et al. [17] found that $46 \%$ had NAFLD and $12.2 \%$ had NASH in a cohort of active duty personnel and their families. They also found a higher prevalence of NAFLD and NASH among patients with diabetes (74 and $22.2 \%$, respectively) in this cohort [17]. Because of the need for liver biopsy to confirm a NASH diagnosis [1], prevalence estimates for NASH in the general population seem to be underestimated.

Together, these studies suggest that because of the increasing prevalence of obesity and underdiagnosing of related conditions such as NAFLD and NASH, there is a need for more accurate diagnosis and proper management of NAFLD. However, awareness and knowledge about NAFLD and NASH continues to be a concern that directly impacts diagnosing practices, and therefore limits our ability to accurately estimate the prevalence of NAFLD and NASH. Several recent studies have examined awareness and knowledge of NAFLD and/or NASH among different groups of healthcare workers. An Italian study of 56 general practitioners revealed $70 \%$ underestimated the prevalence of NAFLD, and when asked only $65 \%$ could indicate the definition of NAFLD was fatty degeneration of hepatocytes in patients with an altered glucose or lipid metabolism [12]. Wieland et al. showed that physicians, particularly PCPs, and specialists other than gastroenterologists and hepatologists within a primarily urban setting within the western United States had a low rate of recognition of NAFLD as a clinically relevant disease regarding its propensity for progression to chronic liver disease and cirrhosis and its associated morbidity and mortality [9]. They also showed that diagnostic and management approaches to patients with NAFLD were highly variable and deviated significantly from practice guidelines, indicating an overreliance on aminotransferase levels specifically to screen for NAFLD. Finally, they suggested that providers underutilized data-driven treatment modalities and also had low rates of referral to gastroenterology and hepatology subspecialists when 
presented with a patient with multiple metabolic risk factors and a high likelihood of having NASH histology.

In a more recent study among various specialists in a metropolitan area of Brisbane, Australia, Bergqvist et al. suggested that $75 \%$ underestimated the prevalence of NAFLD in the general population and $89 \%$ in high-risk populations, $57 \%$ considered alcohol consumption to be strongly associated with NAFLD, 60 \% believed simple steatosis was associated with increased liver related mortality, $66 \%$ thought NASH could be diagnosed with liver imaging, and $71 \%$ made no referrals to GIs/Heps for suspected NAFLD (due in part to the local healthcare system) [10]. Finally, the most recent study by Said et al. in 2013 among physicians in Wisconsin showed lack of confidence in PCPs' knowledge about NAFLD and its management was the top barrier in treating patients with NAFLD. They also showed that, even among physicians treating high risk populations (e.g., obese, diabetics), more than half of primary care practitioners did not screen for NAFLD. The majority (84 \%) also underestimated prevalence of NAFLD in the general and obese population [11].

To obtain a better understanding of awareness of and familiarity with NAFLD and NASH within an unselected group of primary care physicians within the United States, we developed and administered a nation-wide survey to primary care physicians and specialists. Additionally, we aimed to understand how levels of familiarity related to (1) current management of patients with $\mathrm{NASH}$, (2) future plans for self-management or referral of patients with NASH, (3) and specific screening and diagnosing practices for NASH.

\section{Methods}

\section{Survey design}

A national online survey was developed and administered. United States licensed physicians were invited by mail to participate in a $15 \mathrm{~min}$, online national survey. Given the purpose of this study was to determine the current level of physician awareness, familiarity, and practices in the diagnosis and management of NAFLD and NASH, the topic of the survey mentioned on the invitation was generalized to various liver conditions. Awareness of NAFLD and NASH was calculated among all physicians who entered the screener prior to having any knowledge of the purpose of the study, aside from the invitation of the study specifying it was a "national study about various conditions". Specifically, physicians were first asked which of 11 different liver conditions they were aware of, including NAFLD, NASH, seven other liver conditions, and two sham conditions (included as a quality check). Respondents who indicated they were aware of either NAFLD and/or NASH and indicated they were willing to provide accurate responses to questions about their professional experiences qualified for the remainder of the survey. It was assumed that participation in this survey was random and represented basic interest and knowledge in liver conditions. No physician data were excluded from analysis based on their response to the screener questions.

\section{Ethics, consent and permissions}

Physicians were offered an industry-standard honorarium for their time to complete the survey. By opting into to the survey, the respondents provided consent to use their anonymized responses to the survey questions. The study did not collect protected private health information, and it was deemed unnecessary to undergo IRB review according to national regulations $[18,19]$. All survey research conducted for this article was done in accordance with the ethics practices outlined by the Council of American Survey Research Organizations (CASRO) [18].

\section{Survey and data collection}

The survey was live from May 6, 2015 to June 4, 2015, and was comprised of 35 quantitative and qualitative questions. Quantitative questions asked about the following topics: awareness and routine screening of NAFLD and NASH (among various other liver conditions), the level of familiarity with the differences between NAFLD and $\mathrm{NASH}$, current volume of patients managed for NAFLD and NASH, as well as detailed diagnosing and referral practices. Additional questions included awareness of new therapies in development for NASH and what they have heard about each. Qualitative questions asked about perceived differences between NAFLD and NASH, how physicians routinely screen for NASH and what specifically prompts them to refer patients to other physicians for NASH treatment, and how they currently manage their patients with NASH. The survey also contained a short demographic section that asked them to provide their gender, the number of years in practice, and the location and the setting of their practice.

\section{Data analysis}

The individual identities of physician survey respondents were blinded to the study authors. All survey data were analyzed in the aggregate. Responses to the closed questions were analyzed quantitatively. All respondents who qualified for each qualitative question provided comments, because these sections of the survey were mandatory. Responses to the open-ended questions were coded into pre-selected categories before analysis. A response that addressed multiple categories was counted as multiple comments. SPSS Version 20 was used. All continuous 
variables were analyzed using Student's $t$ test. All categorical variables for which the expected cell frequencies were greater than five were analyzed using the Pearson Chi square test. A Fisher's exact test was used whenever the expected cell frequencies were five or less. P values of less than 0.05 were considered significant.

\section{Results}

\section{Survey population and responding physician} demographics

A total of 8119 physicians who specialized in family or internal medicine (hereafter referred to as PCPs), 5920 gastroenterologists, and 238 hepatologists (hereafter referred to as specialists) were randomly selected from the universe of 250,838 PCPs, 14,125 gastroenterologists, and 238 hepatologists, based on the CMS National Plan and Provider Enumeration System. Out of the 354 physicians who entered the screener, 348 completed the screener. Of these 348, 344 identified themselves within our specialties of interest: 185 were PCPs, 128 were gastroenterologists, 31 were hepatologists, and 4 were specialties other than those of interest. A total of 152 PCPs, 121 gastroenterologists, and 29 hepatologists completed the screener and the remainder of the survey. All physicians who completed the screener questions qualified for the survey. This includes an additional 42 physicians (33 PCPs, 7 gastroenterologists, and 2 hepatologists) who completed the screener, but had not completed the survey at the time the data were analyzed. A comparison of the regional distribution of responding physicians (Table 1) was made with the regional distribution of the universe of PCPs and specialists, as determined by the region by state distribution designated by the Census Bureau. No significant difference between the regional breakout of the PCPs in our sample and that of the universe of PCPs was found $(\mathrm{P}=0.080)$. Similarly, no difference between the regional breakout of the specialists in our sample and that of the universe of specialists was found $(\mathrm{P}=0.502)$.

At the time of the survey, the responding PCPs had been practicing for a mean of 14.4 years $(S D=10.96)$. PCPs primarily worked in a group practice (41\%), and the majority practiced in either urban (32\%) or suburban (44\%) locations. Only $28 \%$ of the PCPs were affiliated with an academic institution (Table 2). A total of 150 specialists (121 gastroenterologists and 29 hepatologists) completed the survey. The specialists were in practice for a mean of 16.7 years $(\mathrm{SD}=10.69)$, and $126(84 \%)$ were male.

Compared with PCPs, more specialists were male $(\mathrm{P}<0.001)$, but no difference in length of time in practice was detected between the two groups $(\mathrm{P}=0.087$, Table 2). Differences in practice setting $(\mathrm{P}<0.001)$ and practice location $(\mathrm{P}<0.001)$ were identified. More specialists worked in a group practice $(62 \%)$ and fewer worked in clinics (1\%). Fewer specialists than PCPs practiced in rural areas (6 vs. $24 \%$, respectively).

\section{Awareness of and familiarity with NAFLD and NASH}

Awareness of NAFLD and NASH was calculated among all physicians who entered the screener prior to having any knowledge of the purpose of the study, aside from the invitation of the study specifying it was a "national study about various conditions". Specifically, physicians were first asked which of 11 different liver conditions they were aware of, including NAFLD, NASH, seven other liver conditions, and two sham conditions. Among the 344 physicians within our specialties of interest who completed the screener, $100 \%$ indicated they were aware of NAFLD, NASH, or both. Respondents who indicated they were aware of NAFLD and NASH were then asked to indicate their familiarity with the differences

Table 1 Regional distribution of licensed physicians within the United States and who participated in the online survey

\begin{tabular}{|c|c|c|c|c|c|c|}
\hline \multirow[t]{2}{*}{ Census region } & \multicolumn{2}{|l|}{ PCP } & \multirow[t]{2}{*}{$\mathbf{P}$} & \multicolumn{2}{|c|}{ Specialists } & \multirow[t]{2}{*}{$\mathbf{P}$} \\
\hline & $\begin{array}{l}\text { Sample } \\
\mathrm{n}=152\end{array}$ & $\begin{array}{l}\text { PCP universe } \\
n=250,838\end{array}$ & & $\begin{array}{l}\text { Sample } \\
n=150\end{array}$ & $\begin{array}{l}\text { Universe } \\
n=14,363\end{array}$ & \\
\hline New England & $8(6)$ & $16,470(7)$ & 0.080 & $13(9)$ & $1017(7)$ & $P=0.502$ \\
\hline Middle Atlantic & $26(17)$ & $35,366(14)$ & & $31(21)$ & $2601(18)$ & \\
\hline East North Central & $26(17)$ & $41,295(16)$ & & $21(15)$ & $2012(14)$ & \\
\hline West North Central & $17(11)$ & $18,616(7)$ & & $5(3)$ & $893(6)$ & \\
\hline South Atlantic & $37(24)$ & $46,588(19)$ & & $37(24)$ & $2858(20)$ & \\
\hline East South Central & $5(3)$ & $12,067(5)$ & & $5(3)$ & $746(5)$ & \\
\hline West South Central & $11(7)$ & $23,723(9)$ & & $15(9)$ & $1350(9)$ & \\
\hline Mountain & $4(3)$ & $16,932(7)$ & & $7(5)$ & $793(6)$ & \\
\hline Pacific & $18(12)$ & $39,781(16)$ & & $16(11)$ & $2093(15)$ & \\
\hline
\end{tabular}

Numbers indicated number of responding physicians within the sample (percentage of total responding physicians in the sample), and number of licensed physicians (percentage of total physicians) within each region 
Table 2 Demographics of physician responders

\begin{tabular}{lccc}
\hline & $\begin{array}{l}\text { PCPs } \\
\mathbf{n = 1 5 2}\end{array}$ & $\begin{array}{l}\text { Specialists } \\
\mathbf{n = 1 5 0}\end{array}$ & $\mathbf{P}$ \\
\hline Gender & $102(67)$ & $126(84)$ & \\
Male & $50(33)$ & $24(16)$ & \\
Female & $14.4(<1-50)$ & $16.7(1-59)$ & 0.087 \\
Length of time in practice & & & \\
$\quad$ (mean years, range) & & & $<0.001$ \\
Practice setting & $36(24)$ & $31(21)$ & \\
Hospital-based & $29(19)$ & $24(16)$ & \\
Solo private practice & $62(41)$ & $93(62)$ & \\
Group practice & $25(16)$ & $2(1)$ & \\
$\quad$ Clinic & & & $<0.001$ \\
Practice location & $49(32)$ & $65(43)$ & \\
$\quad$ Urban & $67(44)$ & $76(51)$ & \\
Suburban & $36(24)$ & $9(6)$ & \\
$\quad$ Rural & $43(28)$ & $51(34)$ & 0.284 \\
Affiliated with academic institution & & & \\
\hline
\end{tabular}

Numbers indicate the number of respondents (percentage of total respondents)

between NAFLD and NASH. Among these, $49 \%$ of the PCPs chose either not familiar or unaware of differences between NAFLD and NASH. The majority of specialists (88\%) selected extremely or very familiar with the differences between NAFLD and NASH. $11 \%$ reported they were somewhat familiar with the differences, and $1 \%$ indicated that they were not familiar with or unaware of these differences (Table 3). The rest indicated they were somewhat familiar with the differences. Compared with PCPs, specialists were significantly more likely to report they were familiar with the differences between NAFLD and NASH $(\mathrm{P}<0.001)$. More specialists reported that they routinely screened for NASH (71 vs. $53 \%$; $\mathrm{P}<0.001$ ) and NAFLD (77 vs $56 \%$; $<0.001$, Table 3 ). There was no effect of length of time in practice on familiarity between PCPs and specialists.

Table 3 Physician awareness of the differences between NAFLD and NASH and current screening practices

\begin{tabular}{lccc}
\hline & $\begin{array}{l}\text { PCPs } \\
\mathbf{n = 1 5 2}\end{array}$ & $\begin{array}{l}\text { Specialists } \\
\mathbf{n = 1 5 0}\end{array}$ & $\mathbf{P}$ \\
\hline Awareness of NAFLD/NASH & & & \\
Familiar (extremely/very familiar) & $28(18)$ & $132(88)$ & $<0.001$ \\
Somewhat familiar & $51(33)$ & $16(11)$ & \\
Not familiar/unaware of NASH or NAFLD 73(49) & $2(1)$ & \\
Current screening practices & & & \\
Routinely screen for NASH & $80(53)$ & $107(71)$ & $<0.001$ \\
Routinely screen for NAFLD & $85(56)$ & $115(77)$ & $<0.001$ \\
\hline
\end{tabular}

Numbers indicate the number of respondents (percentage of total respondents)
When asked to specifically describe the differences between NAFLD and NASH, more PCPs than specialists reported that they thought NAFLD and NASH were the same disease ( 9 vs. $0 \%, \mathrm{P}=0.001$ ). PCPs were more likely than specialists to provide generalized descriptions of the differences between NAFLD and NASH (38 vs. $7 \%, \mathrm{P}<0.001)$. Three types of responses were classified as general descriptions of the differences. These included (1) those that only mentioned NASH was more severe than NAFLD; (2) those that mentioned vague differences in the fat deposition, whether correct or incorrect (e.g., "Steatohepatitis is a disease; fatty liver is a result of cholesterol," "One is caused by alcohol; one by too much fat in the liver"); and (3) those that mentioned only that NASH involved a rise in liver enzymes.

Compared to PCPs, specialists reported more specific differences in histologic features (51 vs. $16 \%, \mathrm{P}<0.001$, Table 4). Such responses included (1) those that mentioned the need for a liver biopsy to differentiate; (2) those that indicated the presence of ballooning in NASH; and (3) those that reported differences in histological features such as the levels of fibrosis, scarring, and/or cirrhosis. More specialists (69 \%) than PCPs (24\%) indicated that NAFLD involved no liver inflammation $(\mathrm{P}<0.001)$. Of note, eight PCPs (5\% of all PCPs), which included five PCPs who claimed to be familiar with $\mathrm{NASH}$, and $2 \%$ of specialists reported excessive use of alcohol as a key characteristic of NASH. Finally, $33 \%$ of PCPs and only $1 \%$ of the specialists reported that they were not sure of the differences between NAFLD and NASH ( $<<0.001$, Table 4$)$.

We examined the physician-defined differences based on their reported familiarity with NAFLD and NASH. Of those 79 PCPs who reported being familiar (hereafter referred to as familiar PCPs) with the differences between NAFLD and NASH in the multiple choice question, 5 (6\%) reported that they were unsure of those differences when asked to define the differences. Of 73 PCPs who were not familiar with the differences or unaware of NAFLD or NASH (hereafter referred to as unfamiliar PCPs), 22 (30\%) could provide generalized differences between the two (Table 5). Familiar PCPs were more likely than unfamiliar PCPs to correctly indicate that histologic features $(\mathrm{P}<0.001)$ and inflammation $(\mathrm{P}<0.001)$ were associated with NASH and not NAFLD (Table 5).

\section{Diagnostic practices}

When asked about their diagnostic practices, 50 (34\%) PCPs indicated that they diagnosed patients with NASH (Table 6), and 9 (18\%) of those could identify histological features when asked to recall the differences between NAFLD and NASH. Additionally, 18 (36 \%) PCPs reported that they were not sure of any difference. In contrast 146 specialists were diagnosing patients with 
Table 4 Coded, physician-defined differences between NAFLD and NASH

\begin{tabular}{|c|c|c|c|}
\hline Coded responses & $\begin{array}{l}\text { PCPs } \\
n=152\end{array}$ & $\begin{array}{l}\text { Specialists } \\
n=150\end{array}$ & $\mathbf{P}$ \\
\hline Generalized differences (NET) & $57(38)$ & $10(7)$ & $<0.001$ \\
\hline NASH is more severe than NAFLD & $33(22)$ & $7(5)$ & $<0.001$ \\
\hline General differences in fat deposition & $10(7)$ & $1(1)$ & 0.005 \\
\hline NASH characterized by a rise in liver enzymes & $19(13)$ & $2(1)$ & $<0.001$ \\
\hline Thought they were the same & $13(9)$ & $0(0)$ & $<0.001$ \\
\hline NASH associated with excess alcohol intake & $8(5)$ & $3(2)$ & 0.081 \\
\hline NAFLD involves no inflammation of the liver & $45(30)$ & $101(67)$ & $<0.001$ \\
\hline There is no rise in liver function tests associated with NAFLD, but there is with NASH & $15(10)$ & $11(7)$ & 0.432 \\
\hline Both NASH and NAFLD can be associated with a rise in liver function tests & $7(5)$ & $5(3)$ & 0.199 \\
\hline Increase of fat in the liver associated with both & $32(21)$ & $41(27)$ & 0.202 \\
\hline Histologic features (NET) & $24(16)$ & $77(51)$ & $<0.001$ \\
\hline NASH is diagnosed via a liver biopsy & $5(3)$ & $21(14)$ & $<0.001$ \\
\hline Presence of ballooning indicates NASH & $0(0)$ & $10(7)$ & $<0.001$ \\
\hline Differences in levels of fibrosis/scarring/cirrhosis indicates NASH & $19(13)$ & $45(30)$ & $<0.001$ \\
\hline NASH involves damage to the liver & $21(14)$ & $17(11)$ & 0.515 \\
\hline Not sure of the differences & $50(33)$ & $2(1)$ & $<0.001$ \\
\hline
\end{tabular}

Numbers indicate the number of respondents (percentage of total respondents)

NASH and 75 (51\%) of those could identify differences in histological features of NASH (Table 6), and only 10 (7 \%) reported generalized differences when asked to recall those differences.

Of the 44 responding PCPs who diagnosed patients with NASH, only $25 \%$ of their patients had had a liver biopsy (Table 7). Of the 48 PCPs who had NASH patients that were diagnosed or referred by another physician, $44 \%$ of their patients had had a liver biopsy. Those responding PCPs whose patients were diagnosed by another physician indicated that NASH diagnosis was confirmed by using noninvasive techniques, mainly ultrasound (74 \%) and computed tomography scan (27\%, Table 7). Of the 150 specialists, 143 (97\%) said they diagnosed NASH (Table 7). Of these 143 specialists, $61 \%$ of their patients had had a liver biopsy, and $63 \%$ of their patients without a liver biopsy were not recommended one (Table 7). Among the 32 PCPs and 76 specialists who personally diagnosed at least one patient by a method other than a liver biopsy, significantly more PCPs (94\%) than specialists $(50 \%)$ reported relying solely on imaging and/or liver function tests as a primary method to diagnose NASH $(\mathrm{P}<0.001$, Table 8$)$. Only specialists reported using FibroScan/transient elastography, and/or FibroSure as a primary method to diagnose NASH $(\mathrm{P}<0.001)$.

We examined the physician-defined differences based on whether the respondent routinely screened for NASH (Table 9). Of those 80 PCPs who routinely screened, 20 (25\%) reported that they were unsure of the differences between NAFLD and NASH, and $6(8 \%)$ reported that the diseases were the same. PCPs reported more generalized differences $(\mathrm{P}<0.001)$ and specialists reported more histological differences $(\mathrm{P}<0.001)$.

When asked about what prompts physicians to screen for NAFLD and NASH, specialists were more likely than PCPs to screen patients with diabetes for NAFLD and NASH $(\mathrm{P}=0.001$, Table 10$)$. PCPs were more likely than specialists to screen patients with abdominal pain $(\mathrm{P}=0.001)$.

\section{Management practices}

At the time of taking the survey, responding PCPs were managing an average of 8.5 patients with confirmed $\mathrm{NASH}$, while unfamiliar PCPs were managing an average of 3.7 patients with confirmed NASH. Specialists were managing an average of 32.2 patients with confirmed NASH (Table 11). Physicians were asked to include only patients for whom they were the primary physician making treatment decisions to manage NASH. Of the respondents, $70 \%$ of PCPs and $98 \%$ of specialists were currently managing NASH and/or NAFLD patients (Table 12). More specialists than PCPs reported using vitamin $\mathrm{E}$ and metformin to treat current NASH patients ( $\mathrm{P}<0.001$ and $\mathrm{P}=0.019$, respectively). In addition, $21 \%$ of the PCPs indicated that they would continue to manage all of their new NASH patients.

We then compared management practices for PCPs based on familiarity with NASH (Table 13). At the time of the survey, $58 \%$ (42/73) of unfamiliar PCPs were managing patients with NAFLD or NASH. Despite lack of familiarity with the differences between NAFLD and NASH, unfamiliar PCPs were as likely as familiar PCPs to report 
Table 5 Coded, physician-defined difference between NASH and NAFLD based on PCP familiarity with the NAFLD and NASH differences

\begin{tabular}{|c|c|c|c|}
\hline Coded responses & $\begin{array}{l}\text { Unfamiliar PCPs } \\
n=73\end{array}$ & $\begin{array}{l}\text { Familiar PCPs } \\
\mathrm{n}=79\end{array}$ & $\mathbf{P}$ \\
\hline Generalized differences (NET) & $22(30)$ & $35(44)$ & 0.071 \\
\hline NASH is more severe than NAFLD & $11(15)$ & $22(28)$ & 0.076 \\
\hline General differences in fat deposition & $2(3)$ & $8(10)$ & 0.051 \\
\hline NASH characterized by a rise in liver enzymes & $10(14)$ & $8(10)$ & 0.496 \\
\hline Thought they were the same & $13(18)$ & $0(0)$ & $<0.001$ \\
\hline NASH is associated with excess alcohol intake & $3(4)$ & $5(6)$ & 0.239 \\
\hline NAFLD involves no inflammation of the liver & $11(15)$ & $34(43)$ & $<0.001$ \\
\hline Rise in liver function tests is associated with NASH and not NAFLD & $6(8)$ & $9(11)$ & 0.512 \\
\hline Rise in liver function tests is associated with both NASH and NAFLD & $2(3)$ & $5(6)$ & 0.183 \\
\hline Increase of fat in the liver associated with both & $5(7)$ & $27(34)$ & $<0.001$ \\
\hline Histologic features (NET) & $3(4)$ & $21(27)$ & $<0.001$ \\
\hline NASH is diagnosed via a liver biopsy & $0(0)$ & $5(6)$ & 0.036 \\
\hline Presence of ballooning indicates NASH & $0(0)$ & $0(0)$ & N/A \\
\hline Differences in levels of fibrosis/scarring/cirrhosis indicates NASH & $3(4)$ & $16(20)$ & 0.002 \\
\hline NASH involves damage to the liver & $4(6)$ & $17(22)$ & 0.003 \\
\hline Not sure of the differences & $45(62)$ & $5(6)$ & $<0.001$ \\
\hline
\end{tabular}

Numbers indicate the number of respondents (percentage of total respondents)

Physician may have responded with more than one coded response

Table 6 Current diagnostic practices of physicians and coded differences as defined by physicians who diagnose NASH

\begin{tabular}{|c|c|c|c|}
\hline & $\begin{array}{l}\text { PCPs } \\
n=152\end{array}$ & $\begin{array}{l}\text { Specialists } \\
n=150\end{array}$ & $\mathbf{P}$ \\
\hline \multicolumn{4}{|l|}{ Does the physician diagnose NASH? } \\
\hline Yes & $50(34)$ & $146(97)$ & $<0.001$ \\
\hline Coded responses from physicians who diagnose NASH & $n=50$ & $n=146$ & \\
\hline Generalized differences (NET) & $21(42)$ & $10(7)$ & $<0.001$ \\
\hline NASH is more severe than NAFLD & $13(26)$ & $7(5)$ & $<0.001$ \\
\hline General differences in fat deposition & $3(6)$ & $1(1)$ & 0.048 \\
\hline NASH characterized by a rise in liver enzymes & $6(12)$ & $2(1)$ & 0.004 \\
\hline Thought they were the same & $6(12)$ & $0(0)$ & $<0.001$ \\
\hline NASH associated with excess alcohol intake & $2(4)$ & $3(2)$ & 0.272 \\
\hline NAFLD involves no inflammation of the liver & $12(24)$ & $100(69)$ & $<0.001$ \\
\hline Rise in liver function tests is associated with NASH and not NAFLD & $5(10)$ & $11(8)$ & 0.192 \\
\hline Rise in liver function tests is associated with both NASH and NAFLD & $4(8)$ & $5(3)$ & 0.122 \\
\hline Increase of fat in the liver is associated with both NASH and NAFLD & $9(18)$ & $41(28)$ & 0.158 \\
\hline Histologic features (NET) & $9(18)$ & $75(51)$ & $<0.001$ \\
\hline NASH is diagnosed via a liver biopsy & $1(2)$ & $20(14)$ & 0.012 \\
\hline Presence of ballooning indicates NASH & $0(0)$ & $10(7)$ & 0.048 \\
\hline Differences in levels of fibrosis/scarring/cirrhosis indicates NASH & $8(16)$ & $45(31)$ & 0.042 \\
\hline NASH involves damage to the liver & $5(10)$ & $16(11)$ & 0.208 \\
\hline Not sure of the differences & $18(36)$ & $1(1)$ & $<0.001$ \\
\hline
\end{tabular}

Numbers indicate the number of respondents (percentage of total respondents)

The results in italics above are the individual items that qualify to be counted towards the corresponding NET categories above them. In other words, physicians who mentioned more than one of the items in italics is counted only once in the NET category above it 
Table 7 Aggregate patient-level responses by physicians and mean number of patients seen by physicians who either diagnosed or did not diagnose NASH

\begin{tabular}{|c|c|c|c|}
\hline & PCPs & Specialists & $\mathbf{P}$ \\
\hline \multicolumn{4}{|l|}{ Does the responding physician diagnose NASH? } \\
\hline Yes & $n=44$ & $n=143$ & \\
\hline Patients diagnosed with liver biopsy & $25 \%(4.8)$ & $61 \%(16.3)$ & 0.001 \\
\hline Patients diagnosed without liver biopsy & $75 \%(14.5)$ & $39 \%(10.5)$ & 0.352 \\
\hline No & $n=48$ & $\mathrm{n}=79$ & \\
\hline Patients had liver biopsy & $44 \%(4.1)$ & $43 \%(5.8)$ & 0.320 \\
\hline Patients did not have liver biopsy & $42 \%(3.9)$ & $48 \%(6.5)$ & 0.265 \\
\hline Unknown biopsy status & $14 \%(1.3)$ & $8 \%(1.3)$ & 0.875 \\
\hline $\begin{array}{l}\text { What percentage of patients who did not have a liver biopsy had their } \\
\text { diagnosis for NASH confirmed primarily using the following method(s)? }\end{array}$ & $n=32$ & $n=76$ & \\
\hline Ultrasound & $74 \%(14.8)$ & $66 \%(13.1)$ & 0.754 \\
\hline CT scan & $27 \%(5.3)$ & $20 \%(4.0)$ & 0.386 \\
\hline MRI & $3 \%(0.6)$ & $13 \%(2.6)$ & 0.101 \\
\hline Proton magnetic resonance spectroscopy & $2 \%(0.4)$ & $2 \%(0.3)$ & 0.914 \\
\hline Other & $2 \%(0.5)$ & $19 \%(3.8)$ & 0.152 \\
\hline What is the main reason for lack of liver biopsy? & $n=32$ & $n=76$ & \\
\hline Patient refusal & $51 \%(10.2)$ & $33 \%(6.5)$ & 0.384 \\
\hline Had alternate reason & $5 \%(1.0)$ & $4 \%(0.8)$ & 0.797 \\
\hline Not recommended a liver biopsy & $44 \%(8.7)$ & $63 \%(12.4)$ & 0.394 \\
\hline
\end{tabular}

Number of responding physicians ( $\mathrm{n}$ ) indicated patient specific data (percentage of patients within practice and mean number of patients within each category)

Table 8 The primary method for diagnosing patients with NASH who did not have a liver biopsy

\begin{tabular}{|c|c|c|c|}
\hline Primary method to confirm NASH other than liver biopsy & $\begin{array}{l}\text { PCPs } \\
n=32\end{array}$ & $\begin{array}{l}\text { Specialists } \\
n=76\end{array}$ & $\mathbf{P}$ \\
\hline Mentioned only imaging/liver function tests & $30(94)$ & $38(50)$ & $<0.001$ \\
\hline Ultrasound & $15(47)$ & $21(28)$ & 0.073 \\
\hline CT scan & $4(13)$ & $10(13)$ & 1.000 \\
\hline MRI & $0(0)$ & $6(8)$ & 0.176 \\
\hline Sonogram & $1(3)$ & $1(1)$ & 0.507 \\
\hline Mentioned any method other than imaging/liver function tests & $2(6)$ & $38(50)$ & $<0.001$ \\
\hline Serologic studies (unspecified) & $1(3)$ & $12(16)$ & 0.103 \\
\hline FibroScan/Transient elastography, and/or fibrosure & $0(0)$ & $21(28)$ & $<0.001$ \\
\hline Biochemical tests/risk factors (unspecified) & $1(3)$ & $7(9)$ & 0.432 \\
\hline Ferritin & $0(0)$ & $1(1)$ & 1.000 \\
\hline MRCP & $0(0)$ & $1(1)$ & 1.000 \\
\hline Too vague & $0(0)$ & $5(7)$ & 0.319 \\
\hline
\end{tabular}

that they will manage new NASH patients $(\mathrm{P}=0.514)$. When asked what symptoms would prompt a PCP to refer some patients to other physicians, $67 \%(39 / 58)$ indicated they would refer for worsening laboratory values, liver function tests or abnormal transaminases.

Awareness of therapies in the pipeline for treating NASH When asked about their knowledge of drugs for treating $\mathrm{NASH}$ that are in the development pipeline, more specialists than PCPs were aware of any drug in development (61 vs $36 \%, P<0.001$, Table 14). Fewer than $50 \%$ of the specialists were aware of obeticholic acid while only $12 \%$ of PCPs were aware of it.

\section{Discussion}

Our data suggest significant gaps in physician awareness and familiarity with NAFLD and NASH in the United States. Nearly half of the responding PCPs within our 
Table 9 Coded, physician-defined differences between NAFLD and NASH based on those who said they routinely screen for NASH

\begin{tabular}{|c|c|c|c|}
\hline & $\begin{array}{l}\text { PCPs } \\
n=80\end{array}$ & $\begin{array}{l}\text { Specialists } \\
\mathrm{n}=107\end{array}$ & $\mathbf{P}$ \\
\hline Generalized differences (NET) & $31(39)$ & $9(8)$ & $<0.001$ \\
\hline NASH is more severe than NAFLD & $21(26)$ & $6(6)$ & $<0.001$ \\
\hline General differences in fat deposition & $4(5)$ & $1(1)$ & 0.094 \\
\hline NASH characterized by a rise in liver enzymes & $9(11)$ & $2(2)$ & 0.007 \\
\hline Thought they were the same & $6(8)$ & $0(0)$ & 0.005 \\
\hline NASH associated with excess alcohol intake & $7(9)$ & $3(3)$ & 0.056 \\
\hline NAFLD involves no inflammation of the liver & $29(36)$ & $70(65)$ & $<0.001$ \\
\hline Rise in liver function tests associated with NASH but not NAFLD & $8(10)$ & $8(8)$ & 0.542 \\
\hline Rise in liver function tests is associated with both NASH and & $12(15)$ & $13(12)$ & 0.571 \\
\hline Increase of fat in the liver associated with both & $20(25)$ & $31(29)$ & 0.546 \\
\hline Histologic features (NET) & $17(21)$ & $50(47)$ & $<0.001$ \\
\hline NASH is diagnosed via a liver biopsy & $5(6)$ & $16(15)$ & 0.033 \\
\hline Presence of ballooning indicates NASH & $0(0)$ & $8(8)$ & 0.010 \\
\hline Differences in levels of fibrosis/scarring/cirrhosis indicates NASH & $12(15)$ & $30(28)$ & 0.48 \\
\hline NASH involves damage to the liver & $14(18)$ & $11(10)$ & 0.151 \\
\hline Not sure of the differences & $20(25)$ & $2(2)$ & $<0.001$ \\
\hline
\end{tabular}

Numbers indicate the number of respondents (percentage of total respondents)

Table 10 Main reasons to screen for NASH given by physicians who routinely screen for NAFLD or NASH

\begin{tabular}{lccc}
\hline & PCPs & Specialists & P \\
& $\mathbf{n = 8 0}$ & $\mathbf{n}=107$ & \\
\hline NASH associated with excess alcohol use & $7(9)$ & $3(3)$ & 0.056 \\
Ruling out other conditions & $3(4)$ & $8(8)$ & 0.148 \\
Fatigue & $4(5)$ & $3(3)$ & 0.222 \\
Abnormal labs & $63(79)$ & $78(73)$ & 0.358 \\
Abdominal pain (RUQ) & $19(24)$ & $7(7)$ & 0.001 \\
Diabetes & $35(44)$ & $73(68)$ & 0.001 \\
Metabolic Syndrome & $10(13)$ & $20(19)$ & 0.254 \\
Ultrasound/CT/Imaging & $33(41)$ & $49(46)$ & 0.536 \\
Liver biopsy & $8(10)$ & $46(43)$ & $<0.001$ \\
Obesity & $18(23)$ & $38(36)$ & 0.055 \\
\hline
\end{tabular}

Numbers indicate the number of respondents (percentage of total respondents) study reported being unfamiliar with the differences between NAFLD and NASH, and $58 \%$ of PCPs who were unfamiliar with these differences indicated that they were managing patients with NASH or NAFLD. In addition, these unfamiliar PCPs were just as likely as familiar PCPs to manage new NASH patients.

Because a liver biopsy is required for the diagnosis of $\mathrm{NASH}$ according to the current practice guideline [1], current prevalence estimates of NASH of 2-5\% may be low; not all patients may be willing to undergo a liver biopsy for confirmation. One prospective cohort study found the prevalence of NASH to be $12.2 \%$ among its 328 participants and $29.9 \%$ among the 134 patients with positive ultrasound for fatty liver [17]. Others reported that surveyed physicians other than hepatologists underestimate the prevalence of $[9-11,20]$. This apparent lack

Table 11 Mean number of patients with confirmed or suspected NAFLD or NASH being managed by physicians

\begin{tabular}{|c|c|c|c|}
\hline & $\begin{array}{l}\text { PCPs } \\
n=152\end{array}$ & $\begin{array}{l}\text { Unfamiliar PCPs } \\
n=73\end{array}$ & $\begin{array}{l}\text { Specialists } \\
n=149^{a}\end{array}$ \\
\hline Confirmed NASH & 8.51 & 3.71 & 32.2 \\
\hline NAFLD with suspected NASH (not confirmed) & 10.9 & 6.0 & 41.5 \\
\hline NAFLD with no NASH (confirmed) & 7.94 & 3.2 & 42.1 \\
\hline NAFLD with no suspected NASH & 17.81 & 12.0 & 65.2 \\
\hline Suspected NAFLD & 24.19 & 11.9 & 78.3 \\
\hline
\end{tabular}

a One gastroenterologist who mentioned treating 1000 NAFLD with suspected NASH patients, and 800 NAFLD with no suspected NASH patients was excluded 
Table 12 Management practices of physicians currently treating or expecting to treat new patients with NASH

\begin{tabular}{|c|c|c|c|}
\hline & PCPs & Specialists & $\begin{array}{l}\text { P } \\
\text { (PCPs/ } \\
\text { Specs) }\end{array}$ \\
\hline Does the physician currently manage patients with NASH and/or NAFLD? & $n=152$ & $n=150$ & \\
\hline Yes & $106(70)$ & $147(98)$ & $<0.001$ \\
\hline Coded current management & $n=70$ & $n=144$ & \\
\hline Vitamin $\mathrm{E}$ & $6(9)$ & $86(60)$ & $<0.001$ \\
\hline Hepatitis vaccination & $8(11)$ & $9(6)$ & 0.189 \\
\hline Milk thistle & $0(0)$ & $4(3)$ & 0.202 \\
\hline Statin & $5(7)$ & $8(6)$ & 0.208 \\
\hline Pentoxyphyllin & $0(0)$ & $2(1)$ & 0.452 \\
\hline Metformin & $1(1)$ & $15(10)$ & 0.011 \\
\hline Actigall & $0(0)$ & $2(1)$ & 0.452 \\
\hline Will the physician manage new NASH patients? & $n=148$ & $n=150$ & $<0.001$ \\
\hline Yes & $31(21)$ & $129(86)$ & \\
\hline Yes, but will refer some new patients & $62(42)$ & $18(12)$ & \\
\hline No, will refer all new patients & $55(37)$ & $3(2)$ & \\
\hline
\end{tabular}

Numbers indicate the number of respondents (percentage of total responding)

Table 13 Current management practices of physicians based on familiarity with the NAFLD and NASH differences

\begin{tabular}{|c|c|c|c|}
\hline & Unfamiliar PCPs & Familiar PCPs & $\mathbf{P}$ \\
\hline Does the physician currently manage patients with NASH or NAFLD? & $n=73$ & $n=79$ & 0.002 \\
\hline Yes, NASH and/or NAFLD patients & $42(58)$ & $64(81)$ & \\
\hline Will the physician manage new NASH patients? & $n=69$ & $\mathrm{n}=79$ & 0.514 \\
\hline Yes & $13(19)$ & $18(23)$ & \\
\hline Yes, but will refer some new patients & $27(39)$ & $35(44)$ & \\
\hline No, will refer all new patients & $29(42)$ & $26(33)$ & \\
\hline
\end{tabular}

Numbers indicate the number of respondents (percentage of total responding)

of understanding of the prevalence of NAFLD suggests a limited knowledge of NAFLD, and as a result screening for NAFLD and NASH may be limited. Only about half of the PCPs in our study reported that they routinely screen for NAFLD and NASH.

Williams et al. reported that the prevalence of advanced NASH in untreated patients was $2.7 \%$ (nine patients) and extrapolated their data to the general population, suggesting that more than two million middle-aged adults in the United States may have undiagnosed advanced NASH [17]. Since patients with advanced NASH have a poor prognosis [21], PCPs, who are typically the first point of contact within the United States healthcare system, not only need to be aware of NAFLD and NASH, but also need to know how to effectively manage these patients to limit disease progression.

While our study did not specifically ask PCPs if they were aware of the guideline, we did ask questions that
Table 14 Physician awareness of therapies in development for treating NASH

\begin{tabular}{lccr}
\hline & $\begin{array}{l}\text { (a) } \\
\text { PCPs } \\
\mathbf{n = 1 5 2}\end{array}$ & $\begin{array}{l}\text { Specialists } \\
\mathbf{n = 1 5 0}\end{array}$ & \multicolumn{1}{c}{} \\
& & \\
\hline Aware of any drug in development & $54(36)$ & $91(61)$ & $<0.001$ \\
Obeticholic acid (OCA) & $18(12)$ & $72(48)$ & $<0.001$ \\
Aramchol & $7(5)$ & $22(15)$ & 0.002 \\
GR-MD-02 & $5(3)$ & $13(9)$ & 0.028 \\
Simtuzumab & $9(6)$ & $31(21)$ & $<0.001$ \\
GFT505 & $4(3)$ & $10(7)$ & 0.056 \\
Proscysbi & $1(1)$ & $10(7)$ & 0.004 \\
Emricasan & $1(1)$ & $8(5)$ & 0.016 \\
GCS-100 & $1(1)$ & $2(1)$ & 0.374 \\
MN-001 & $1(1)$ & $5(3)$ & 0.090 \\
LUM001 & $2(1)$ & $3(2)$ & 0.312 \\
\hline
\end{tabular}

Numbers indicate the number of respondents (percentage of total responding) 
allowed us to indirectly assess adherence to the practice guideline. Of the surveyed PCPs who personally diagnose $\mathrm{NASH}, 75 \%$ of their patients had not been diagnosed by using the results of a liver biopsy. Additionally, PCPs who were treating referral patients for NASH reported that $42 \%$ of their patients had not had a liver biopsy (Table 7). Our data suggest that practicing PCPs within the United States may not be using the current practice guideline to diagnose NAFLD and NASH, and these data further support the work of others $[4,22]$ who suggested the current practice guideline may not provide a practical approach for PCPs to diagnose NASH. Although divergence from the guidelines might be an indicator of the lack of alternative solutions to a liver biopsy, the substantial difference in practices between PCPs and specialists in light of the large gap in knowledge between PCPs and specialists indicate this is likely a result of the limited knowledge among PCPs. PCPs might choose to continue managing patients due to the belief that there is no better alternative a specialist can offer.

At the time of our survey, unfamiliar PCPs were managing about four patients with confirmed NASH (Table 11). In addition, the proportion of responses from unfamiliar PCPs was not different from the proportion of responses from the familiar PCPs when asked about their future plans to manage new NASH patients. These data are somewhat surprising, because this indicates an apparent overconfidence of the physicians who manage NASH patients but do so without NASH-specific knowledge.

PCPs were less likely than specialists to use vitamin E to treat patients with NASH; however, we did not ask whether these PCPs only used vitamin E in patients with liver biopsy-confirmed NASH. Furthermore, some specialists were using metformin even though no data support its use for treating NASH [1]. These data indicate that these responding physicians appear to not be following the practice guideline for treating NASH. Although this could indicate lack of awareness of such guidelines, it is likely more of a reflection of the skepticism with guidelines as it relates to treatments, given their overall lack of efficacy. As expected, more specialists than PCPs did have some knowledge about the drugs in development to treat NASH.

One practical approach to narrowing the gap in knowledge between PCPs and specialists starts with establishing a program for accreditation of centers with special expertise in NAFLD-related liver conditions, particularly NASH, to raise the overall quality of care and outcomes in patients with this life-threatening disease. This program should be multidisciplinary and global in order to capitalize on the highly interrelated nature of this condition to its underlying risk factors. Efforts to create this program can mirror those of The Pulmonary
Hypertension Association's Scientific Leadership Council's effort in establishing the PHA-Accredited PH Care Centers (PHCC) in order to raise the overall quality of care and outcomes in patients with pulmonary hypertension $(\mathrm{PH})$, in particular pulmonary arterial hypertension (PAH) [23]. Similar to NAFLD, PH is a highly prevalent condition that is often confused with related conditions, with PAH affecting only a small subset of patients with a very different prognosis, similar to NASH. Like NASH, PAH was often misdiagnosed due to the need for a right heart catheterization in order to accurately determine the diagnosis. Right heart catheterizations, however, presented a challenge for many physicians and patients due to similar reasons liver biopsies will remain a challenge to perform among all patients with suspected NAFLD or NASH. Such expert center would lead the process of coming to a consensus on what biomarkers can be used in diagnosing and in evaluating drugs in development. Once revised guidelines are achieved based on these recommendations, it would serve to accredit physicians or centers in the proper diagnosing of NAFLD-related liver conditions. Additionally, it will be important for these programs to then collaborate with the highly structured existing centers, such as diabetes education centers, that are aimed to maximize knowledge and proper treatment of underlying risk factors of NAFLD-related liver conditions. Our study had three limitations. First, while low, our response rates are similar to other mailed surveys. Second, the abilities of the physicians to recall specific patient data may have influenced their responses. Third, the survey did not specifically ask about awareness of the practice guideline for NAFLD and NASH, therefore, we cannot comment on the specific knowledge of the practice guideline in relation to the diagnosis and management of NASH.

\section{Conclusions}

The awareness of and familiarity with the differences between NAFLD and NASH by surveyed PCP in the United States is limited. While specialists were generally more knowledgeable about these differences, not all specialists were following the diagnosis and treatment guideline. Therefore, more education for PCPs and specialists is needed to ensure NAFLD and NASH are appropriately diagnosed and managed.

\section{Abbreviations}

NAFLD: nonalcoholic fatty liver disease; NASH: nonalcoholic steatohepatitis; NHANES III: Third National Health and Nutrition Examination Survey; PCP: primary care physician.

\section{Authors' contributions}

All of the authors contributed to the conception and design of the study and analysis methods. SPB and DG collected and analyzed the data. SPB, DG, and 
MS drafted the manuscript. AC supervised all aspects of the study. All of the authors contributed to interpreting the data, critically revised the manuscript for important intellectual content and approved the final version of the submitted manuscript. All authors read and approved the final manuscript.

\section{Acknowledgements}

Under the direction of the authors, Kristina Wasson-Blader, PhD, drafted the initial version of the manuscript and provided editorial assistance.

\section{Competing interests}

The authors declare that they have no competing interests.

Received: 8 December 2015 Accepted: 22 February 2016 Published online: 11 March 2016

\section{References}

1. Chalasani N, Younossi Z, Lavine JE, Diehl AM, Brunt EM, Cusi K, Charlton $M$, Sanyal AJ. The diagnosis and management of non-alcoholic fatty liver disease: practice guideline by the American Association for the Study of Liver Diseases, American College of Gastroenterology, and the American Gastroenterological Association. Hepatology. 2012;55(6):2005-23.

2. Wong RJ, Aguilar M, Cheung R, Perumpail RB, Harrison SA, Younossi ZM, Ahmed A. Nonalcoholic steatohepatitis is the second leading etiology of liver disease among adults awaiting liver transplantation in the United States. Gastroenterology. 2015;148(3):547-55.

3. Vernon G, Baranova A, Younossi ZM. Systematic review: the epidemiology and natural history of non-alcoholic fatty liver disease and non-alcoholic steatohepatitis in adults. Aliment Pharmacol Ther. 2011;34(3):274-85.

4. Nascimbeni F, Pais R, Bellentani S, Day CP, Ratziu V, Loria P, Lonardo A. From NAFLD in clinical practice to answers from guidelines. J Hepatol. 2013;59(4):859-71.

5. Arulanandan A, Loomba R. Noninvasive testing for NASH and NASH with advanced fibrosis: are we there yet? Curr Hepatology Rep. 2015:14:109-18.

6. Rinella ME, Lominadze Z, Loomba R, Charlton M, Neushwander-Tetri BA, Caldwell SH, Kowdley K, Harrison SA. Practice patterns in NAFLD and NASH: real life differs from published guidelines. Therap Adv Gastroenterol. 2015;9(1):4-12

7. Brunt EM. Nonalcoholic steatohepatitis. Semin Liver Dis. 2004;24(1):3-20

8. Ratziu V, Goodman Z, Sanyal A. Current efforts and trends in the treatment of NASH. J Hepatol. 2015;62(1 Suppl):S65-75.

9. Wieland AC, Quallick M, Truesdale A, Mettler P, Bambha KM. Identifying practice gaps to optimize medical care for patients with nonalcoholic fatty liver disease. Dig Dis Sci. 2013;58(10):2809-16.

10. Bergqvist CJ, Skoien R, Horsfall L, Clouston AD, Jonsson JR, Powell EE. Awareness and opinions of non-alcoholic fatty liver disease by hospital specialists. Intern Med J. 2013;43(3):247-53.
11. Said A, Gagovic V, Malecki K, Givens ML, Nieto FJ. Primary care practitioners survey of non-alcoholic fatty liver disease. Ann Hepatol. 2013;12(5):758-65.

12. Grattagliano I, D’Ambrosio G, Palmieri VO, Moschetta A, Palasciano G Portincasa P. Improving nonalcoholic fatty liver disease management by general practitioners: a critical evaluation and impact of an educational training program. J Gastrointestin Liver Dis. 2008;17(4):389-94.

13. Harrison SA, Okada SY, Su CA, Paulson M, J.D. B, Sanyal AJ. U.S. Physician survey of current practices in the diagnosis and treatment of nonalcoholic steatohepatitis (NASH): program and abstracts of the 65th annual meeting of the American Association for the Study of Liver Diseases (AASLD). Hepatology 2014; 60(1):Suppl Abstract 630.

14. Lominadze Z, Harrison SA, Charlton M, Loomba R, NeuschwanderTetri BA, Caldwell SH, Kowdley K, Rinella ME. Survey of diagnostic and treatment patterns of NAFLD and NASH in the United States: real life practices differ from published guidelines. Program and abstracts of the 65th Annual Meeting of the American Association for the Study of Liver Diseases (AASLD) Abstract 838. Hepatology. 2014; 60:Suppl.

15. Kallman JB, Arsalla A, Park V, Dhungel S, Bhatia P, Haddad D, Wheeler A Younossi ZM. Screening for hepatitis $B, C$ and non-alcoholic fatty liver disease: a survey of community-based physicians. Aliment Pharmacol Ther. 2009;29(9):1019-24.

16. Lazo M, Hernaez R, Bonekamp S, Kamel IR, Brancati FL, Guallar E, Clark JM. Non-alcoholic fatty liver disease and mortality among US adults: prospective cohort study. BMJ. 2011;343:d6891.

17. Williams CD, Stengel J, Asike MI, Torres DM, Shaw J, Contreras M, Landt $\mathrm{CL}$, Harrison SA. Prevalence of nonalcoholic fatty liver disease and nonalcoholic steatohepatitis among a largely middle-aged population utilizing ultrasound and liver biopsy: a prospective study. Gastroenterology. $2011 ; 140(1): 124-31$

18. CASRO. Code of standards and ethics. Accessed at http://www.casro. org/?page=TheCASROCode2014 on 30 Nov 2015.

19. HHS gov. US Department of Health and Human Services. Code of Federal Regulations accessed at http://www.hhs.gov/ohrp/humansubjects/ guidance/45cfr46.html\#46.102 on 30 Nov 2015.

20. Ratziu V, Bellentani S, Cortez-Pinto H, Day C, Marchesini G. A position statement on NAFLD/NASH based on the EASL 2009 special conference. J Hepatol. 2010;53:372-84

21. Dyson JK, Anstee QM, McPherson S. Non-alcoholic fatty liver disease: a practical approach to diagnosis and staging. Frontline Gastroenterol. 2014;5(3):211-8

22. Grattagliano I, Ubaldi E, Napoli L, Marulli CF, Nebiacolombo C, Cottone C, Portincasa P. Utility of noninvasive methods for the characterization of nonalcoholic liver steatosis in the family practice. The "VARES" Italian multicenter study. Ann Hepatol. 2013;12(1):70-7.

23. Pulmonary hypertension association accessed at http://www.phassociation.org/PHCareCenters on 4 Jan 2016

\section{Submit your next manuscript to BioMed Central and we will help you at every step:}

- We accept pre-submission inquiries

- Our selector tool helps you to find the most relevant journal

- We provide round the clock customer support

- Convenient online submission

- Thorough peer review

- Inclusion in PubMed and all major indexing services

- Maximum visibility for your research

Submit your manuscript at www.biomedcentral.com/submit
() Biomed Central 